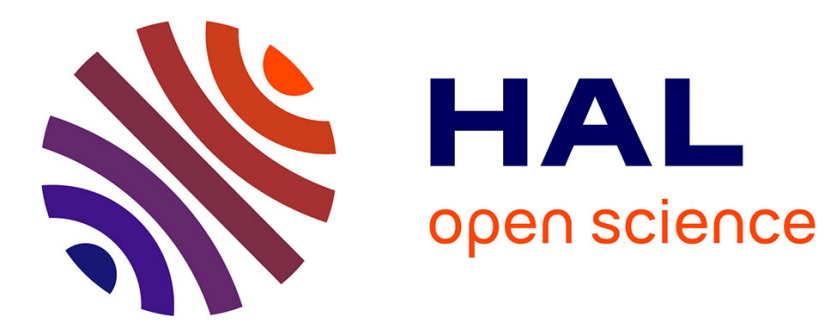

\title{
Characteristic Constraint Modes for Component Mode Synthesis
}

Matthew P Castanier, Yung-Chang Tan, Christophe Pierre

\section{To cite this version:}

Matthew P Castanier, Yung-Chang Tan, Christophe Pierre. Characteristic Constraint Modes for Component Mode Synthesis. AIAA Journal, 2001, 39, pp.1182-1187. 10.1115/1.1338948 . hal01537673

\section{HAL Id: hal-01537673 \\ https://hal.science/hal-01537673}

Submitted on 12 Jun 2017

HAL is a multi-disciplinary open access archive for the deposit and dissemination of scientific research documents, whether they are published or not. The documents may come from teaching and research institutions in France or abroad, or from public or private research centers.
L'archive ouverte pluridisciplinaire HAL, est destinée au dépôt et à la diffusion de documents scientifiques de niveau recherche, publiés ou non, émanant des établissements d'enseignement et de recherche français ou étrangers, des laboratoires publics ou privés. 


\title{
Characteristic Constraint Modes for Component Mode Synthesis
}

\author{
Matthew P. Castanier, Yung-Chang Tan, and Christophe Pierre \\ University of Michigan, Ann Arbor, Michigan 48109-2125
}

\begin{abstract}
A technique is presented for reducing the size of a model generated by the Craig-Bampton method (Craig, R. R., and Bampton, M. C. C., "Coupling of Substructures for Dynamic Analyses," AIAA Journal, Vol. 6, No. 7, 1968, pp. 1313-1319) of component mode synthesis (CMS). An eigenanalysis is performed on the partitions of the CMS mass and stiffness matrices that correspond to the so-called constraint modes. The resultant eigenvectors are referred to as characteristic constraint modes because they represent the characteristic motion of the interface between the component structures. When the characteristic constraint modes are truncated, a CMS model with a highly reduced number of degrees of freedom may be obtained. An example of a cantilever plate is considered. It is shown that relatively few characteristic constraint modes are needed to yield accurate approximations of the lower natural frequencies. Furthermore, this method yields physical insight into the mechanisms of vibration transmission in complex structures, and it provides an excellent framework for the efficient calculation of power flow.
\end{abstract}

\section{Nomenclature}

$=$ stiffness matrix

$=$ stiffness matrix partition

$=$ stiffness matrix partition that has been reduced in both dimensions (rows and columns) by coordinate transformations using selected sets of modes

$=$ mass matrix

$=$ mass matrix partition

$=$ mass matrix partition that has been reduced in both dimensions (rows and columns)

by coordinate transformations using selected sets of modes

$=$ number of physical or modal degrees of freedom (DOF)

$=$ displacements in physical coordinates, for example, finite element DOF

$=$ generalized coordinates of the component mode synthesis model

$=$ generalized coordinates of the reduced-order model

$=$ eigenvalue

$=$ full set of characteristic constraint eigenvectors

$=$ selected set of characteristic constraint eigenvectors

$=$ full set of normal modes for substructure $i$

$=$ selected set of normal modes for substructure $i$

$=$ characteristic constraint eigenvector (in constraint-mode DOF)

$=$ normal mode for substructure $i$

$=$ full set of Craig-Bampton constraint modes ${ }^{2}$

$=$ characteristic constraint mode (in physical coordinates)

$=$ constraint-mode DOF

$=$ characteristic-constraint-mode DOF

\begin{tabular}{|c|c|}
\hline CMS & $=$ component mode synthesis model \\
\hline ROM & $=$ reduced-ordermodel \\
\hline$\alpha$ & $\begin{aligned}= & \text { physical DOF of substructure } 1, \\
& \text { excluding the interface DOF }\end{aligned}$ \\
\hline$\alpha \Gamma, \beta \Gamma$ & $=$ coupling terms between physical $\mathrm{DOF}$ \\
\hline$\Gamma \alpha, \Gamma \beta$ & of a substructure and the interface \\
\hline$\beta$ & $\begin{aligned}= & \text { physical DOF of substructure } 2, \\
& \text { excluding the interface DOF }\end{aligned}$ \\
\hline$\Gamma$ & $\begin{aligned}= & \text { physical DOF of the interface } \\
& \text { between substructures }\end{aligned}$ \\
\hline 1 & $=$ normal-mode $\mathrm{DOF}$ of substructure 1 \\
\hline 2 & $=$ normal-mode DOF of substructure 2 \\
\hline $\begin{array}{l}1 C, 2 C \\
C 1, C 2\end{array}$ & $\begin{array}{l}=\text { coupling terms between normal-mode } \\
\text { and constraint-mode DOF }\end{array}$ \\
\hline $\begin{array}{l}1 \mathcal{C C}, 2 \mathcal{C C} \\
\mathcal{C C} 1, \mathcal{C C} 2\end{array}$ & $\begin{aligned}= & \text { coupling terms between normal-mode } \\
& \text { and characteristic-constraint-mode DOF }\end{aligned}$ \\
\hline
\end{tabular}

\section{Introduction}

W HEN modeling the dynamics of a complex structure, it is often impractical to perform a finite element analysis of the entire structure. In some cases, the finite element model (FEM) of the full structure has so many degrees of freedom (DOF) that a global finite element analysis is impossible due to computermemory constraints or node limits in the software. Also, it is common for component structures to be designed or redesigned independently, which makes it more convenient to perform a separate finite element analysis for each component.

Component mode synthesis (CMS) was developed as a practical and efficient approach to modeling and analyzing the dynamics of the global structure in such circumstances. ${ }^{1,2}$ In CMS, the dynamics of a structure are described by selected sets of normal modes of the individual component structures, plus a set of static vectors that account for the coupling at each interface where component structures are connected. Thus, each component structure is dynamically reduced by a separate modal analysis before being coupled at the system level, yielding savings in finite element costs as well as providing significant order reduction for the CMS model relative to the FEM of the complex structure. For a review of the CMS literature, the reader is referred to the work by $\mathrm{Craig}^{3}$ and Seshu. ${ }^{4}$

One of the most accurate and widely used CMS methods is the Craig-Bampton method. ${ }^{2,5}$ In this method, the component normal modes are calculated with the interface between the component structures held fixed. Attachment at the interface is achieved by a set of constraint modes. A constraint mode shape is the static deflection induced in the structure by applying a unit displacement to one interface DOF while all of the other interface DOF are held fixed. Thus, the motion at the interface is completely described by the constraint modes. However, there is a computational cost associated with these modes because the CMS model must have 
one DOF for each DOF in the interface of the FEM. This means that, whereas dramatic order reduction may be achieved for the individual components (normal-mode DOF), there is no order reduction for the interface (constraint-modeDOF). In fact, if the finite element mesh is sufficiently fine, the size of the CMS model may be dominated by the constraint-modeDOF. This can make the CMS model cumbersome to use, for example, in analysis of large-scale structures with many components or in probabilistic analysis.

In this paper, a new technique is presented for reducing the size of a CMS model by performing an eigenanalysis on the constraintmode partitions of the mass and stiffness matrices. The resultant eigenvectors are called the characteristic constraint $(\mathcal{C C})$ modes. These modes may then be truncated to yield a highly reducedorder model. Therefore, the method presented in this paper offers increased computational efficiency for performing analyses with a CMS model. Furthermore, the $\mathcal{C C}$ modes evidence the characteristic motion of the interface, so that this method provides significant physical insight into the transmission of vibration energy between substructures. In particular, a $\mathcal{C C}$-mode-based model is well suited to the efficient calculation of power flow in complex structures, due to the compact representation of the interface motion.

To the authors' knowledge, the only similar method is that of Bourquin and d'Hennezel., 7 They introduced a fixed-interface CMS method "based on a non-conventional choice of constraint modes tied to the normal modes of the Poincaré-Steklov operator associated with the interface between the substructures. ${ }^{6}$ They called these alternative constraint modes the "coupling modes," and they proposed algorithms for handling the various numerical computations needed to find the coupling modes. ${ }^{7}$ In contrast, the method proposed here is based on the classic Craig-Bampton method ${ }^{2,5}$ and employs a straightforward eigenanalysis to find the characteristic constraint modes. Therefore, this new method is quite simple, both conceptually and computationally.

This paper is organized as follows. In the second section, the Craig-Bampton method of CMS is briefly reviewed, and the constraint modes are discussed. In the third section, the $\mathcal{C C}$ modes are introduced as a means of reducing the size of the CMS model, and the advantages of a $\mathcal{C C}$-mode-based representation are covered. For illustration, traditional constraint modes and $\mathcal{C C}$ modes are compared for two different finite element models of a two-span beam on simple supports. In the fourth section, the new CMS method is applied to an example system of a cantilever plate. The $\mathcal{C C}$ mode shapes are shown, and the accuracy in calculating the natural frequencies with various sets of $\mathcal{C C}$ modes is discussed. This work is summarized in the final section.

\section{Component Mode Synthesis and Constraint Modes}

Consider a FEM of a complex structure that is partitioned into two substructures, that is, component structures. The boundary where the component structures are connected will be referred to as the interface. The group of DOF in the interface will be denoted by $\Gamma$, and the number of interface DOF by $N_{\Gamma}$. The DOF of substructure 1 (2) that are not in $\Gamma$ will be denoted by $\alpha(\beta)$, and the number of these DOF will be denoted by $N_{\alpha}\left(N_{\beta}\right)$. Then the mass and stiffness matrices in finite element coordinates may be partitioned as follows:

$$
\boldsymbol{M}=\left[\begin{array}{ccc}
\boldsymbol{m}_{\Gamma} & \mathbf{0} & \mathbf{0} \\
\mathbf{0} & \boldsymbol{m}_{\alpha} & \mathbf{0} \\
\mathbf{0} & \mathbf{0} & \boldsymbol{m}_{\beta}
\end{array}\right], \quad \boldsymbol{K}=\left[\begin{array}{ccc}
\boldsymbol{k}_{\Gamma} & \boldsymbol{k}_{\Gamma \alpha} & \boldsymbol{k}_{\Gamma \beta} \\
\boldsymbol{k}_{\alpha \Gamma} & \boldsymbol{k}_{\alpha} & \mathbf{0} \\
\boldsymbol{k}_{\beta \Gamma} & \mathbf{0} & \boldsymbol{k}_{\beta}
\end{array}\right]
$$

where subscripts denote the associated DOF and double subscripts indicate coupling terms.

The partitioning scheme of Eq. (1) is chosen for simplicity of presentation. In general, one may choose to have other active DOF, such as points where forcing is applied or where a damper is attached. These DOF would then be grouped with the interface DOF. Also, CMS formulations are usually cast in terms of the substructure matrices. However, for the purposes of this study, it is more straightforward to partition the matrices of the full complex structure into interface and noninterface DOF. Furthermore, only two component structures are considered, but this is not restrictive.

The Craig-Bampton method ${ }^{2}$ of component mode synthesis employs fixed-interface component normal modes, as well as a set of vectors called constraint modes. As mentioned earlier, a constraint mode is the static deflection induced in the structure by applying a unit displacement to one interface DOF while all other interface DOF are held fixed. Therefore, the interface partition of the constraint modes is an identity matrix of dimension $N_{\Gamma}$, and the set of constraint modes $\Psi^{C}$ is of the form

$$
\Psi^{C}=\left[\begin{array}{c}
\boldsymbol{I} \\
\Psi_{\alpha}^{C} \\
\Psi_{\beta}^{C}
\end{array}\right]
$$

where $\Psi_{\alpha}^{C}$ and $\Psi_{\beta}^{C}$ are the static shapes induced in substructures 1 and 2, respectively, by the unit displacements at the interface. These shapes are determined by posing the following statics problem:

$$
\left[\begin{array}{ccc}
\boldsymbol{k}_{\Gamma} & \boldsymbol{k}_{\Gamma \alpha} & \boldsymbol{k}_{\Gamma \beta} \\
\boldsymbol{k}_{\alpha \Gamma} & \boldsymbol{k}_{\alpha} & \mathbf{0} \\
\boldsymbol{k}_{\beta \Gamma} & \mathbf{0} & \boldsymbol{k}_{\beta}
\end{array}\right]\left[\begin{array}{c}
\boldsymbol{I} \\
\Psi_{\alpha}^{C} \\
\boldsymbol{\Psi}_{\beta}^{C}
\end{array}\right]=\left[\begin{array}{l}
\boldsymbol{f} \\
\mathbf{0} \\
\mathbf{0}
\end{array}\right]
$$

where $f$ is a vector of forces that would be needed to impose the successive unit displacements at the interface DOF. The two sets of homogeneous equations in Eq. (3) yield

$$
\Psi_{\alpha}^{C}=-\left(\boldsymbol{k}_{\alpha}\right)^{-1} \boldsymbol{k}_{\alpha \Gamma}, \quad \Psi_{\beta}^{C}=-\left(\boldsymbol{k}_{\beta}\right)^{-1} \boldsymbol{k}_{\beta \Gamma}
$$

Using these solutions, the constraint modes of Eq. (2) are known.

Next, the component normal modes are obtained by performing a modal analysis for each component structure. Because the CraigBampton method uses fixed-interface modes, they are determined from the following eigenvalue problems:

$$
\boldsymbol{k}_{\alpha} \phi_{1}^{\mathcal{N}}=\lambda \boldsymbol{m}_{\alpha} \phi_{1}^{\mathcal{N}}, \quad \boldsymbol{k}_{\beta} \phi_{2}^{\mathcal{N}}=\lambda \boldsymbol{m}_{\beta} \phi_{2}^{\mathcal{N}}
$$

In general, these modes are truncated to reduce the size of the model. For example, if $\Phi_{i}^{\mathcal{N}}$ is the full eigenvector matrix for substructure $i$ and $N_{i}$ modes are selected,

$$
\underbrace{\Phi_{1}^{\mathcal{N}}}_{N_{\alpha} \times N_{\alpha}} \stackrel{\hat{s}^{\mathcal{N}}}{\hat{\Phi}_{1}^{\mathcal{N}}}, \quad \underbrace{\Phi_{2}^{\mathcal{N}}}_{N_{\alpha} \times N_{1}} \stackrel{\underbrace{}_{N_{\beta} \times N_{\beta}}}{\text { select modes }} \underbrace{\hat{\Phi}_{2}^{\mathcal{N}}}_{N_{\beta} \times N_{2}}
$$

Then, by the use of the constraint modes and selected sets of component normal modes, the transformation from finite element coordinates $\boldsymbol{x}$ to CMS generalized coordinates $\boldsymbol{y}$ is

$$
\boldsymbol{x}=\left[\begin{array}{ccc}
\boldsymbol{I} & \mathbf{0} & \mathbf{0} \\
\Psi_{\alpha}^{C} & \hat{\Phi}_{1}^{\mathcal{N}} & \mathbf{0} \\
\Psi_{\beta}^{C} & \mathbf{0} & \hat{\Phi}_{2}^{\mathcal{N}}
\end{array}\right] \boldsymbol{y}
$$

Applying the transformationof Eq. (7), the global mass and stiffness matrices for the CMS model have the form ${ }^{3}$

$$
\boldsymbol{M}_{\mathrm{CMS}}=\left[\begin{array}{ccc}
\boldsymbol{m}_{C} & \boldsymbol{m}_{C 1} & \boldsymbol{m}_{C 2} \\
\boldsymbol{m}_{1 C} & \hat{\boldsymbol{m}}_{1} & \mathbf{0} \\
\boldsymbol{m}_{2 C} & \mathbf{0} & \hat{\boldsymbol{m}}_{2}
\end{array}\right], \quad \boldsymbol{K}_{\mathrm{CMS}}=\left[\begin{array}{ccc}
\boldsymbol{k}_{C} & \mathbf{0} & \mathbf{0} \\
\mathbf{0} & \hat{\boldsymbol{k}}_{1} & \mathbf{0} \\
\mathbf{0} & \mathbf{0} & \hat{\boldsymbol{k}}_{2}
\end{array}\right]
$$

Note that this transformation yields only inertial coupling between the normal modes and the constraint modes.

The number of normal-mode DOF depends, of course, on how many normal modes are selected for each component structure. However, the number of constraint-mode DOF is equal to $N_{\Gamma}$, the number of FEM DOF that are in the interface. Thus, the size of the constraint-mode partitions is determined by the finite element mesh. If there are many finite element nodes in the interface region, then the constraint-mode partitions of the CMS matrices may be 


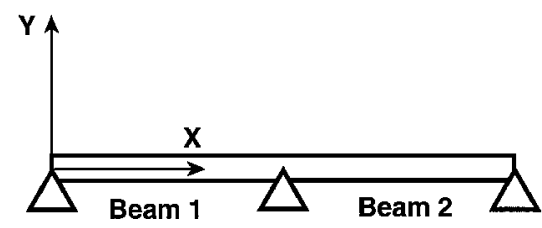

Fig. 1 Two-span beam on simple supports.

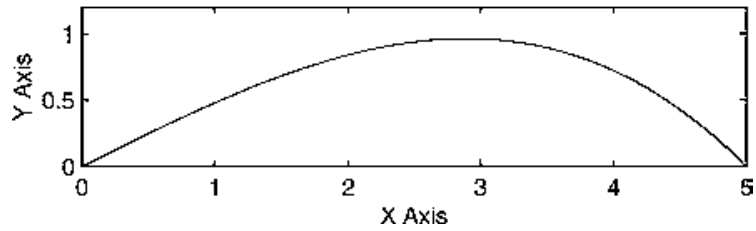

Fig. 2 Single constraint mode for the beam-element FEM.

relatively large. Furthermore, as the number of constraint modes increases, the individual constraint modes may have limited physical meaning.

To illustrate the last point, consider the simply supported twospan beam presented in Fig. 1. This system is treated as a complex structure consisting of two component structures with the interface at the center support. Each span is treated as a substructure, referred to as beam 1 and beam 2. (The modeling of power flow in this system was examined in detail in an earlier work by the authors. ${ }^{8}$ ) Two different finite element models were generated for this system: one consisting of Timoshenko beam elements and a second, more generalFEM consisting of solid elements. All finite element analysis in this study was performed using the commercial code ABAQUS.

For the beam-element FEM, the displacement at the center support is constrained by the pin, so that there is only one (rotational) DOF at the interface. Thus, there is only one constraint mode in the corresponding CMS model. This single constraint mode, which is obtained by imposing a static angular displacement about the center support, is shown in Fig. 2. Only beam 1 is shown. The interface is at the right end.

In contrast, the solid-element FEM has 25 nodes at the interface. Note that one horizontalline of nodes in the center of the interface is fixed to represent a pinned condition. This leaves 20 unconstrained finite element nodes (60 DOF) in the interface of the solid-element FEM, leading to 60 constraint modes in the CMS model. One of these constraintmodes is shown in Fig. 3. Only beam 1 is shown. The interface node at which a unit displacement is imposed is marked by a dot. Significant local deformation is evident in this constraint mode. It can also be seen that the offset of the imposed displacement from the pinned line of nodes leads to bending of the beam in the $X Y$ plane. It is easy to imagine that a linear combination of similar constraint modes could yield a more natural flexural motion, such as that of the lone constraint mode for the beam-element FEM. Furthermore, a complete set of these linear combinations could be defined as a new set of constraint modes that describe the principal ways in which the interfacedeforms. This idea is consideredin detail in the next section.

\section{Modes}

It is now suggested that the number of necessary constraint modes may be reduced by seeking a new set of modes that correspond to more natural physical motion at the interface. This is posed as an eigenvalue problem for the constraint-mode partitions of the CMS matrices:

$$
\boldsymbol{k}_{C} \phi^{\mathcal{C C}}=\lambda \boldsymbol{m}_{C} \phi^{\mathcal{C C}}
$$

Suppose that these eigenvectorsare then truncated, as in a traditional modal analysis:

$$
\underbrace{\Phi^{\mathcal{C C}}}_{N_{\Gamma} \times N_{\Gamma}} \stackrel{\text { select modes }}{\hat{\Phi}^{\mathcal{C C}}}
$$

This selected set of eigenvectors may be used to transform the mass and stiffness matrices to yield a reduced-order model (ROM). The

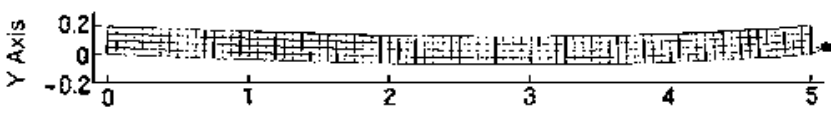

a)

$X$ Axis

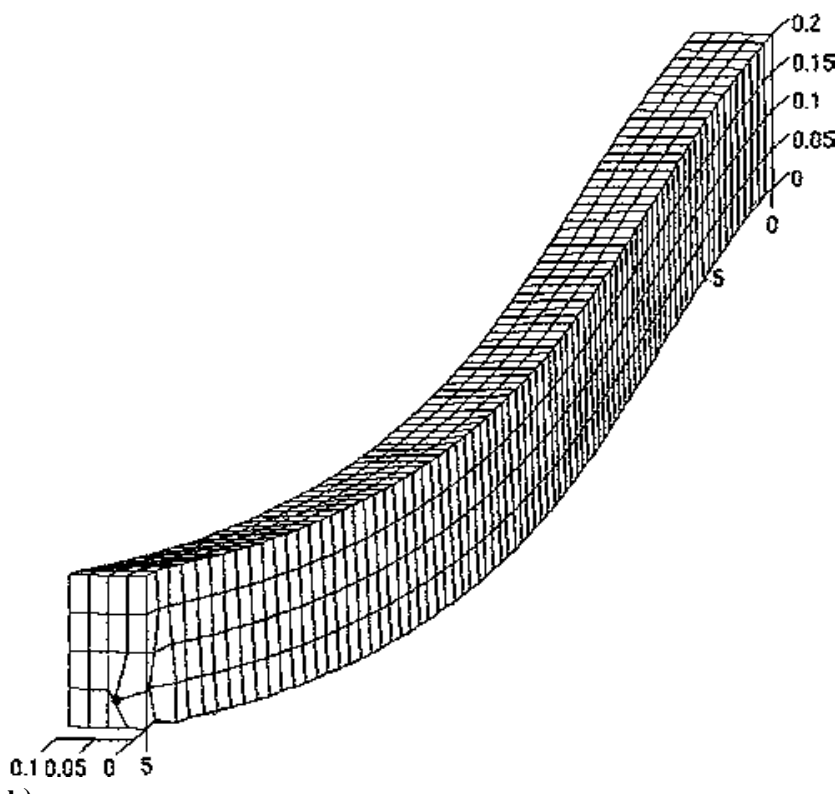

b)

Fig. 3 One of the 60 constraint modes for the solid-element FEM: a) side view of beam 1, as in Fig. 1, with the interface at the right end and b) rotated view of beam 1 with the interface shown in the foreground.

transformation from CMS generalized coordinates $y$ to ROM generalized coordinates $z$ may be defined as

$$
\boldsymbol{y}=\left[\begin{array}{ccc}
\hat{\Phi}^{\mathcal{C C}} & 0 & 0 \\
0 & I & 0 \\
0 & 0 & I
\end{array}\right] z
$$

The mass and stiffness matrices in ROM generalized coordinates are, thus,

$$
\begin{aligned}
\boldsymbol{M}_{\mathrm{ROM}}= & {\left[\begin{array}{ccc}
\hat{\boldsymbol{\Phi}}^{\mathcal{C C}}{ }^{T} \boldsymbol{m}_{C} \hat{\boldsymbol{\Phi}}^{\mathcal{C C}} & \hat{\boldsymbol{\Phi}}^{\mathcal{C}{ }^{T}} \boldsymbol{m}_{C 1} & \hat{\boldsymbol{\Phi}}^{\mathcal{C C}} \boldsymbol{m}_{C 2} \\
\boldsymbol{m}_{1 C} \hat{\boldsymbol{\Phi}}^{\mathcal{C C}} & \hat{\boldsymbol{m}}_{1} & \mathbf{0} \\
\boldsymbol{m}_{2 C} \hat{\boldsymbol{\Phi}}^{\mathcal{C C}} & \mathbf{0} & \hat{\boldsymbol{m}}_{2}
\end{array}\right] } \\
= & {\left[\begin{array}{ccc}
\hat{\boldsymbol{m}}_{\mathcal{C C}} & \hat{\boldsymbol{m}}_{\mathcal{C C} 1} & \hat{\boldsymbol{m}}_{\mathcal{C C} 2} \\
\hat{\boldsymbol{m}}_{1 \mathcal{C C}} & \hat{\boldsymbol{m}}_{1} & \mathbf{0} \\
\hat{\boldsymbol{m}}_{2 \mathcal{C C}} & \mathbf{0} & \hat{\boldsymbol{m}}_{2}
\end{array}\right] } \\
\boldsymbol{K}_{\mathrm{ROM}}= & {\left[\begin{array}{cccc}
\hat{\boldsymbol{\Phi}}^{\mathcal{C C}} \boldsymbol{k}_{C} \hat{\boldsymbol{\Phi}}^{\mathcal{C C}} & \mathbf{0} & \mathbf{0} \\
\mathbf{0} & \hat{\boldsymbol{k}}_{1} & \mathbf{0} \\
\mathbf{0} & \mathbf{0} & \hat{\boldsymbol{k}}_{2}
\end{array}\right]=\left[\begin{array}{ccc}
\hat{\boldsymbol{k}}_{\mathcal{C C}} & \mathbf{0} & \mathbf{0} \\
\mathbf{0} & \hat{\boldsymbol{k}}_{1} & \mathbf{0} \\
\mathbf{0} & \mathbf{0} & \hat{\boldsymbol{k}}_{2}
\end{array}\right] }
\end{aligned}
$$

Now, compared to the mass and stiffness matrices of Eq. (1), the size of every matrix partition has been reduced.

Finally, an eigenvector from Eq. (9) may be transformed from constraint-mode coordinates into finite element coordinates:

$$
\psi^{\mathcal{C C}}=\Psi^{C} \phi^{\mathcal{C C}}
$$

Note that $\psi^{\mathcal{C C}}$ is an eigenvector-based linear combination of constraint modes. Therefore, it captures some characteristic physical motion in the interface region, and accordingly, it is called a characteristic constraint $(\mathcal{C C})$ mode. The $\mathcal{C C}$ modes provide, at least in an approximate sense, the principal modes of deformation for the interface. In fact, the calculation and selection of the $\mathcal{C C}$ modes is essentially a secondary modal analysis, and the benefits are similar 


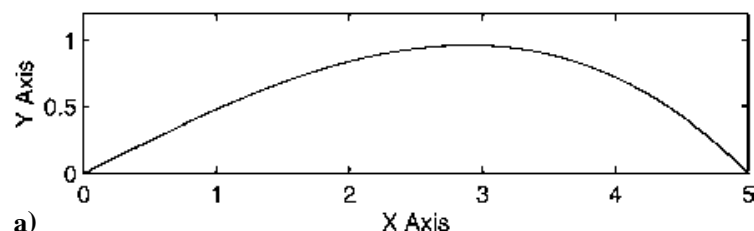

a)

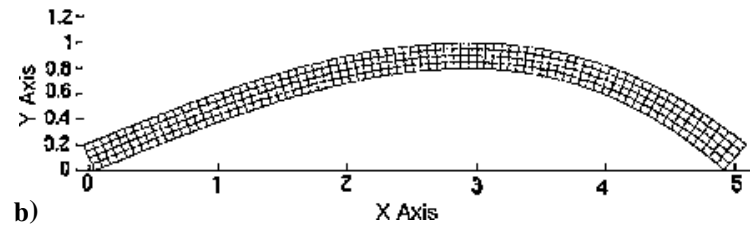

Fig. 4 Comparison of a) single constraint mode for the beam-element FEM and b) first $\mathcal{C C}$ mode for the solid-element FEM.

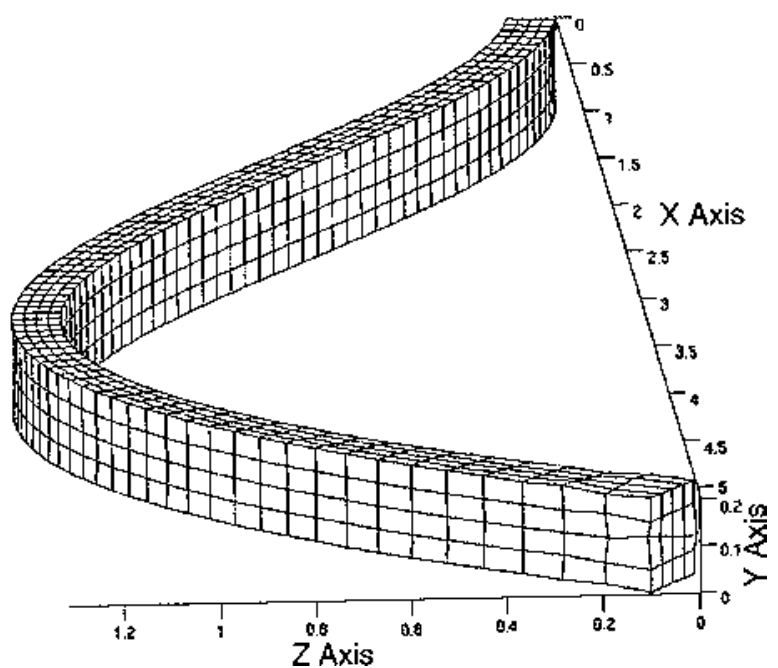

Fig. 5 Second $\mathcal{C}$ mode for the solid-element FEM.

to those of a traditional modal analysis. For example, a refinement of the finite element mesh would lead to an increase in the accuracy of the ROM without requiring an increase in the size of the ROM because the same number of $\mathcal{C C}$ modes could be retained.

Note that, in certain cases, it might be more beneficial to perform an eigenanalysison the full matrices of the CMS model. This would yield a set of system modes for the complex structure. This idea has been investigated by the authors in the context of modeling the vibration of turbomachinery rotors, ${ }^{9,10}$ but it will not be considered here.

To illustrate the characteristic motion that is captured by the $\mathcal{C C}$ modes, the first $\mathcal{C C}$ mode for the solid-element FEM of the beam system is shown in Fig. 4b. Only beam 1 is shown. The interface is at the right end. Note that it appears to be identical to the single constraint mode for the beam-element FEM, which is shown again in Fig. 4a for comparison. It is clear that the first $\mathcal{C C}$ mode captures the $X Y$-plane flexural motion at the interface. The second $\mathcal{C C}$ mode for the solid-elementFEM is shown in Fig. 5. It is apparent that the second $\mathcal{C C}$ mode corresponds to flexural motion in the $X Z$ plane. Only beam 1 is shown. Figure 5 is a rotated view with the interface shown in the foreground.

Thus, depending on the type of motion and frequency range of interest, the $\mathcal{C C}$ modes may be truncated to yield a new CMS model with a greatly reduced number of DOF. The efficiency and accuracy of this method will be investigated in the next section.

\section{Example}

The cantilever plate shown in Fig. 6 is now considered as an example structure. The dimensions and material properties are chosen to be the same as those used by Craig and Bampton ${ }^{2}:$ 2024-T3 aluminum, Young's modulus $E=10.5 \times 10^{6} \mathrm{psi}(72 \mathrm{GPa})$, Poisson's ratio $v=0.33$, density $\rho=0.101 \mathrm{lb} / \mathrm{in}^{3}{ }^{3}\left(2800 \mathrm{~kg} / \mathrm{m}^{3}\right)$, and thickness

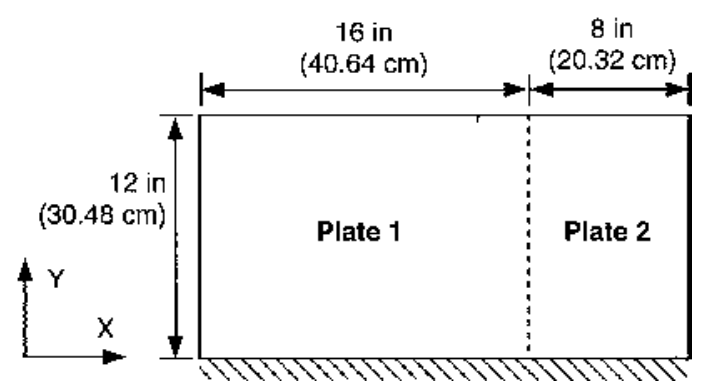

Fig. 6 Cantilever plate partitioned into two component structures, referred to as plate 1 and plate 2 .
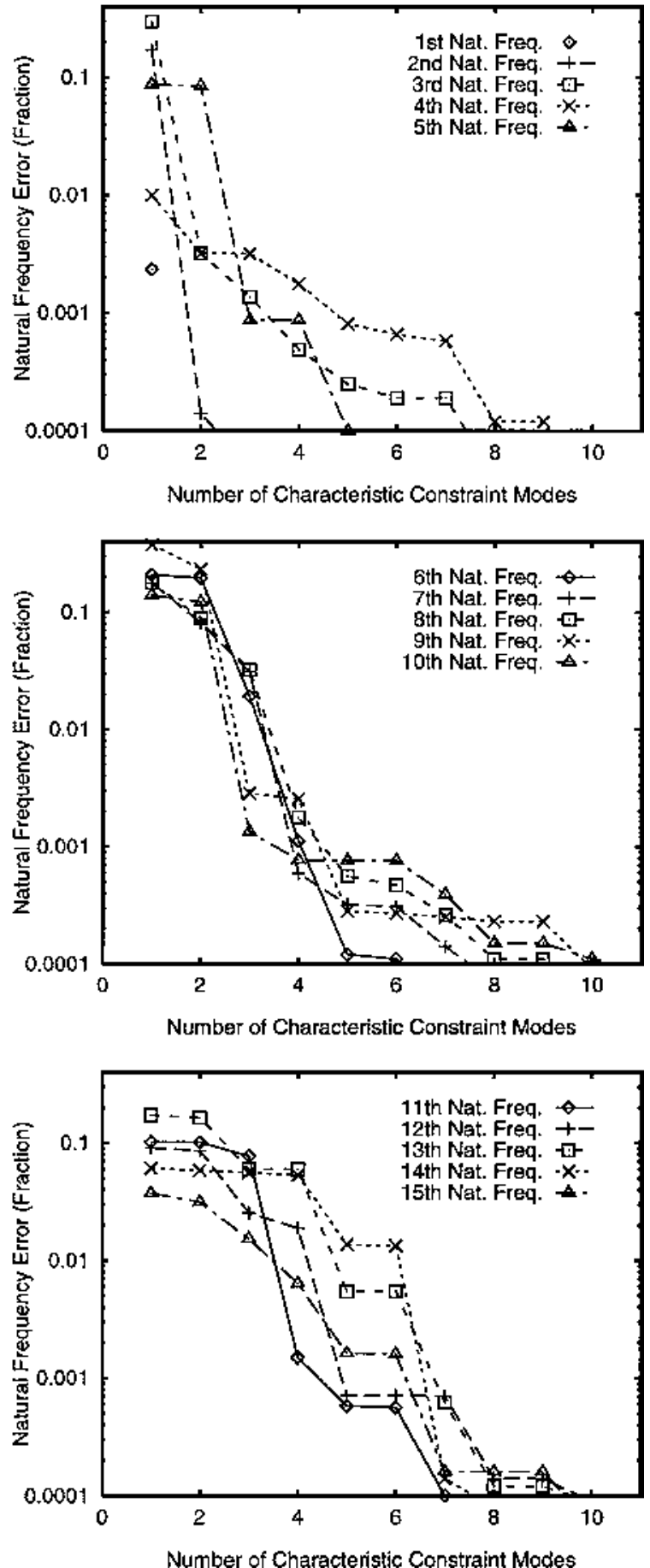

Fig. 7 Error, relative to the 164-DOF CMS model, for the first 15 natural frequencies of the cantilever plate calculated for 10 different ROMs (1-10 retained $\mathcal{C C}$ modes). 
$t=0.125 \mathrm{in} .(0.3175 \mathrm{~cm})$. The plate is considered to consist of two substructures as shown. This partitioning is also the same as that of Ref. 2.

A FEM of this plate was constructed using a $48 \times 24 \times 1$ mesh of ABAQUS shell elements (element S4R, four nodes/element, six $\mathrm{DOF} /$ node). The analytical results for the first 10 natural frequencies of the cantilever plate are given by Gorman, ${ }^{11}$ and these were used as a benchmark. The finite element mesh is sufficiently fine that the first $10 \mathrm{FEM}$ natural frequencies are all within $0.7 \%$ of the analytical results.

A Craig-Bampton CMS model ${ }^{2}$ of the plate was then generated. The first 10 normal modes were retained for each component structure. In addition, there were 144 constraint modes, yielding a CMS model with 164 DOF. For reference, the first 15 natural frequencies of this CMS model are all within $0.9 \%$ of the corresponding FEM natural frequencies.

Next, $\mathcal{C C}$ modes were calculated, and 10 different ROMs were constructed by keeping only the lowest one to $10 \mathrm{CC}$ modes. Because a total of 20 component normal modes were selected for the CMS model, the smallest ROM considered contains 21 DOF and the largest contains 30 DOF.

The error for the first 15 natural frequencies of the cantileverplate is shown vs the number of selected $\mathcal{C C}$ modes in Fig. 7. This error

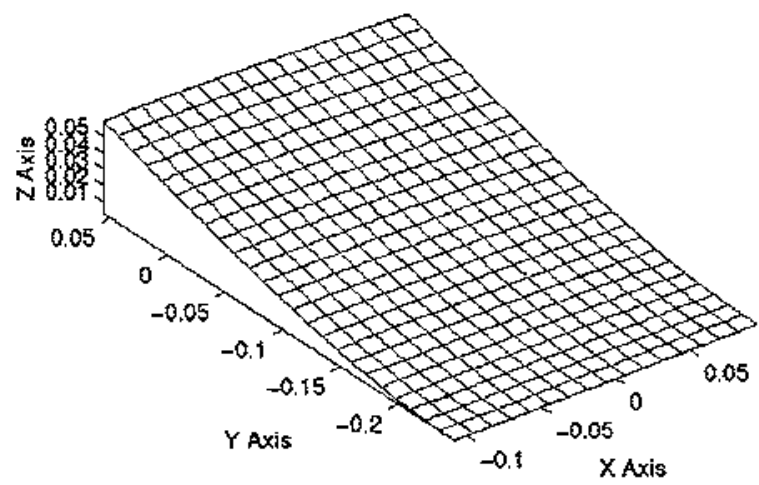

CC Mode 1

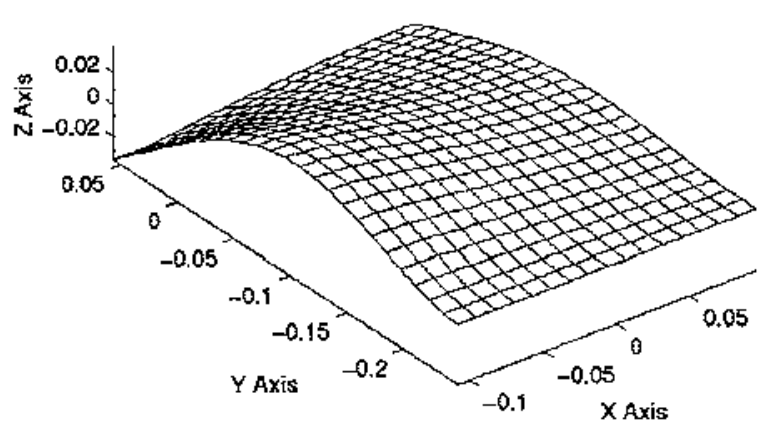

CC Mode 3

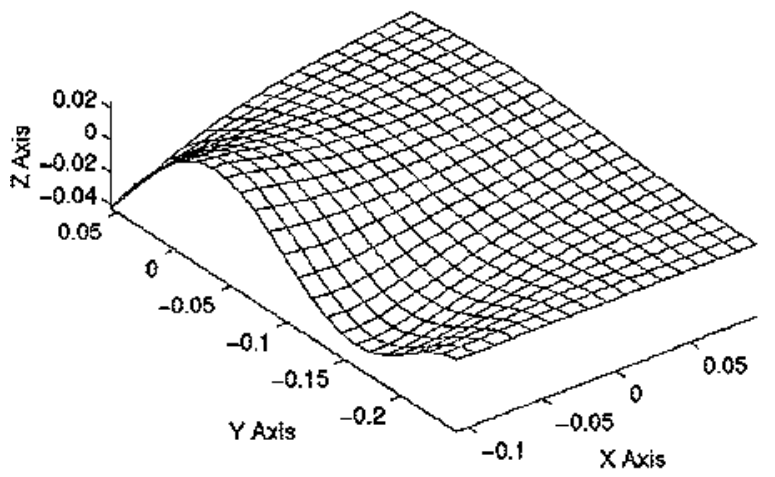

CC Mode 5 is calculated relative to the $164-\mathrm{DOF}$ CMS model because a $\mathcal{C C}$ mode-based model will be no more accurate than its parent CMS model. In fact, if all of the $\mathcal{C C}$ modes are included, no approximation is made. Note that only $10 \mathcal{C C}$ modes are needed to drive the error below $0.01 \%$ for the first 15 natural frequencies of the cantilever plate. Therefore, using the present method, a 30-DOF CMS model is highly accurate relative to the 164-DOF CMS model for the frequency range considered. Note that if the FEM mesh were refined, the full CMS model size would increase. Yet a ROM calculated using the selected set of $\mathcal{C C}$ modes would become more accurate without a penalty of any additional DOF.

Insight into the results presented in Fig. 7 may be gained by comparing the $\mathcal{C C}$ modes to the global modes of the cantileverplate. The first six $\mathcal{C C}$ modes for plate 2 are shown in Fig. 8. (Only plate 2 is shown. Views are rotated such that the interface is seen at the lower left edge, along the $y$ axis.) Consider, for example, the second $\mathcal{C C}$ mode. It is clear that the second $\mathcal{C C}$ mode captures much of the interface-inducedmotion seen in the second and third global modes, which are shown in Fig. 9. This explains why the frequency error decreases by more than two orders of magnitude for these global modes (Fig. 7) when $\mathcal{C C}$ mode 2 is included in the ROM. In Fig. 9, for reference, the interface between plates 1 and 2 is marked by a dark line.

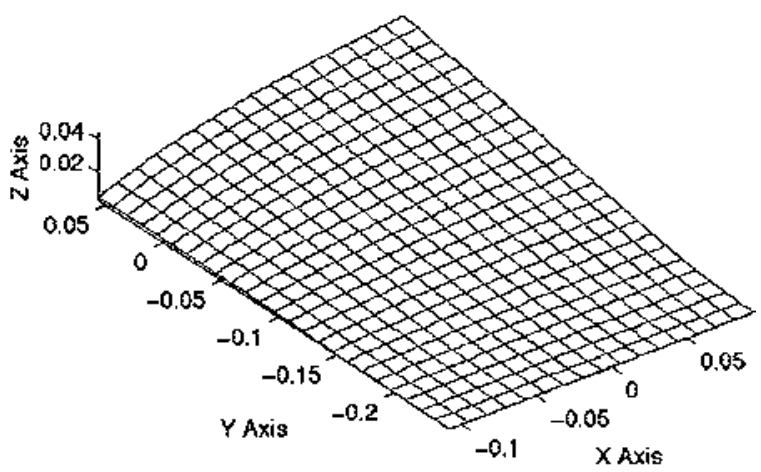

CC Mode 2

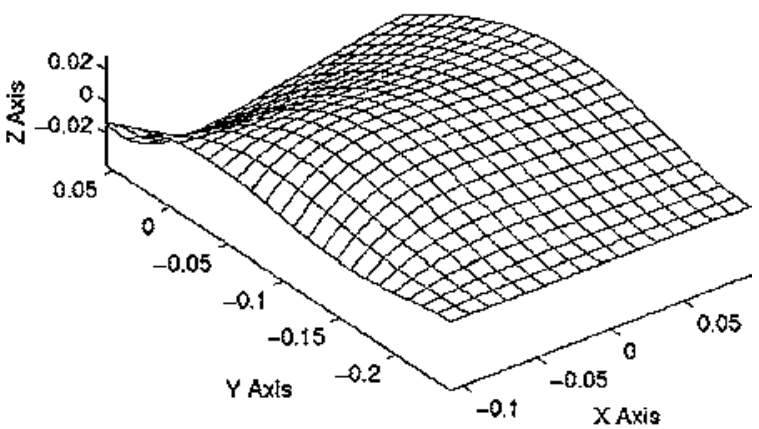

CC Mode 4

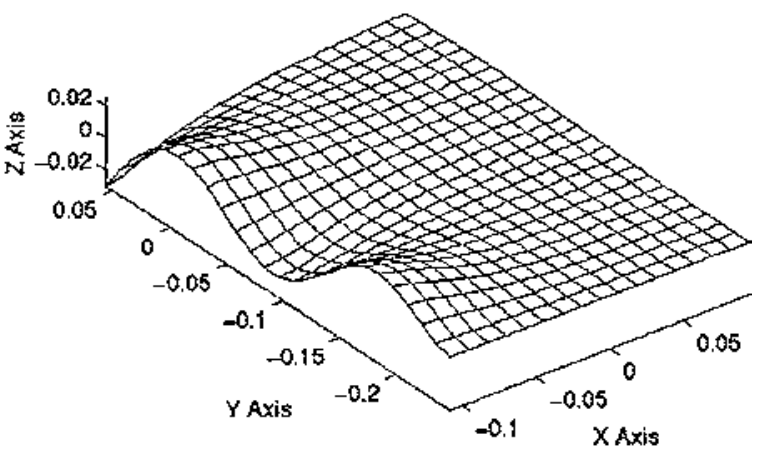

CC Mode 6

Fig. 8 First six characteristic constraint modes for the cantilever plate. 

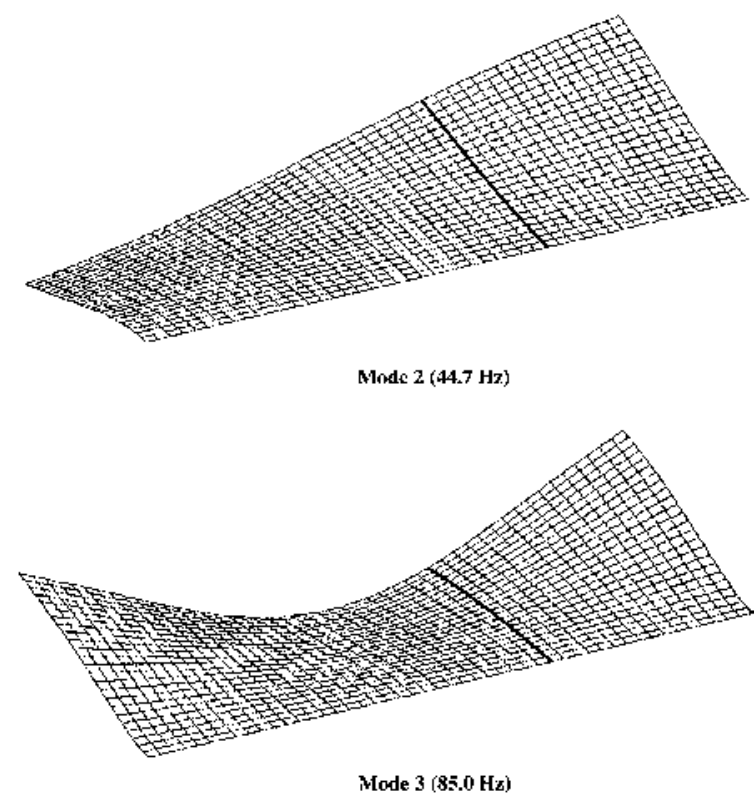

Fig. 9 Second and third global modes for the cantilever plate.

Returning to Fig. 8, note that the wavelength of the interface motion decreases with higher $\mathcal{C C}$ modes, as would be expected. Furthermore, there is an attendant decrease in the influence of the interface motion at the far end of the component structure. Thus, it appears that the lower $\mathcal{C C}$ modes capture most of the global influence of the interface motion. Higher $\mathcal{C C}$ modes, then, would be needed only to model the details of the local interface motion.

\section{Conclusions}

This paper introduceda techniquefor reducing the size of a CraigBampton $\mathrm{CMS}^{2,5}$ model by improving the representation of the interface between component structures. This new method is based on computing $\mathcal{C C}$ modes. The $\mathcal{C C}$ modes are found by performing an eigenanalysis of the partitions of the CMS mass and stiffness matrices that correspond to the Craig-Bampton constraint modes.

It was seen that the $\mathcal{C C}$ modes capture the characteristic motion of the interface, and thus, they may be truncated as if they were traditional modes of vibration. This truncation leads to a highly reduced-orderCMS model. In addition, the $\mathcal{C C}$ modes allow significant insight into the physical mechanisms of vibration transmission between the component structures. This information could be used, for example, to determine the design parameters that have a critical impact on power flow.

The calculation and selection of the $\mathcal{C C}$ modes is essentially a secondary modal analysis. Therefore, the benefits are the same as those of a traditional modal analysis. For instance, refining the finite element mesh would increase the accuracy of a ROM without introducing any additional DOF.

In general, this investigation showed that the use of $\mathcal{C C}$ modes in CMS allows highly efficient modeling of the dynamics of complex structures. Also, this techniqueis capable of providing physical insight into how the vibration energy flows through the structure. Whereas it is believed that this method has wide applicability, it seems especially suited to predicting power flow in complex structures.

\section{References}

${ }^{1}$ Hurty, W. C., "Dynamic Analysis of Structural Systems Using Component Modes," AIAA Journal, Vol. 3, No. 4, 1965, pp. 678-685.

${ }^{2}$ Craig, R. R., and Bampton, M. C. C., "Coupling of Substructures for Dynamic Analyses," AIAA Journal, Vol. 6, No. 7, 1968, pp. 1313-1319.

${ }^{3}$ Craig, R. R., Structural Dynamics: An Introduction to Computer Methods, Wiley, New York, 1981, Chap. 19.

${ }^{4}$ Seshu, P., "Substructuring and Component Mode Synthesis," Shock and Vibration, Vol. 4, No. 3, 1997, pp. 199-210.

${ }^{5}$ Craig, R. R., "Substructure Methods in Vibration," Journal of Mechanical Design, Vol. 117B, June 1995, pp. 207-213.

${ }^{6}$ Bourquin, F., and d'Hennezel, F., "Intrinsic Component Mode Synthesis and Plate Vibrations," Computers and Structures, Vol. 44, No. 1-2, 1992, pp. 315-324.

${ }^{7}$ Bourquin, F., and d'Hennezel, F., "Numerical Study of an Intrinsic Component Mode Synthesis Method," Computer Methods in Applied Mechanics and Engineering, Vol. 97, No. 1, 1992, pp. 49-76.

${ }^{8}$ Tan, Y. C., Castanier, M. P., and Pierre, C., "Approximations of Power Flow Between Two Coupled Beams Using Statistical Energy Methods," Proceedings of the 1998ASME InternationalMechanical Engineering Congress and Exposition, American Society of Mechanical Engineers, Fairfield, NJ, 1998.

${ }^{9}$ Bladh, R., Castanier, M. P., and Pierre, C., "Component-Mode-Based Reduced Order Modeling Techniques for Mistuned Bladed Disks-Part I: Theoretical Models," Journal of Engineering for Gas Turbines and Power, Vol. 123, No. 1, 2001, pp. 89-99.

${ }^{10}$ Bladh, R., Castanier, M. P., and Pierre, C., "Component-Mode-Based Reduced Order Modeling Techniques for Mistuned Bladed Disks-Part II: Application," Journal of Engineering for Gas Turbines and Power, Vol. 123, No. 1, 2001, pp. 100-108.

${ }^{11}$ Gorman, D. J., Free Vibration Analysis of Rectangular Plates, Elsevier, New York, 1982, Chap. 4. 